\title{
A review of the efficacy and safety of several antihyperglycemic medications in the management of cardiovascular disease in diabetes
}

\author{
Mustafa Kinaan ${ }^{1}$, Erin Epstein ${ }^{2}$ and Suzanne Quinn Martinez ${ }^{3 *}$ \\ ${ }^{1}$ Department of Internal Medicine, PGY3, University of Central Florida/ Orlando Veterans Administration Hospital, USA \\ ${ }^{2}$ Department of Physiology, Auburn University, USA \\ ${ }^{3}$ Clinical Associate Professor, University of Central Florida, Division of Endocrinology, Orlando Veterans Administration Hospital, USA
}

\begin{abstract}
Diabetes is a worldwide public health problem affecting millions of people. By 2030, the incidence of diabetes is expected to increase to $4.4 \%$ of the global population, an equivalent of approximately 366 million people. This growth of the "diabetes pandemic" is even more pronounced in certain countries including the United States, which is expected to have a $165 \%$ increase in prevalence by 2050 compared to that in 2000 . Diabetes is a leading cause of morbidity and mortality, which results from microvascular or macrovascular disease. Compared to non-diabetic individuals, diabetics have higher risk of coronary artery disease, cardiac ischemia, cerebrovascular disease, and peripheral vascular disease. Myocardial infarction is nearly 1.8 times more frequent in diabetic patients compared to non-diabetic equivalents. Patients with diabetes also have a high burden of atherosclerotic disease risk factors like hypertension, hyperlipidemia, and obesity. Managing hyperglycemia has been and remains at the center of managing diabetes and preventing its complications. Since 1923 when insulin was first introduced, several hypoglycemic agents have emerged. Experimental and clinical studies have examined the efficacy of these medications in preventing cardiovascular complications and whether a certain drug class has an inherent protective role independent of its hypoglycemic effect. This review will shed light on the evidence regarding the cardiovascular protective role of current hypoglycemic agents.
\end{abstract}

\section{Introduction}

The American Diabetes Association reports that in 201221 million Americans were suffering from diabetes, 8.1 million diabetics remained undiagnosed and another 86 million individuals had prediabetes [1]. Diabetes is the seventh leading cause of death in the United States but may be significantly under-reported. After adjusting for age differences the cardiovascular disease death rate is 1.7 times higher among adults with a diagnosis of diabetes than it is among their non-diabetic counterparts. Myocardial infarction is 1.8 times more frequent and stroke is 1.5 times more frequent in adults with diabetes. Approximately $60 \%$ of non-traumatic lower extremity amputations occur in individuals with diabetes as well. The vascular complications of diabetes are described as microvascular diseases including retinopathy, neuropathy, and nephropathy and macrovascular diseases such as cardiovascular and peripheral vascular disease. And while the microvascular complications certainly contribute to disease morbidity it is the macrovascular disease which affects mortality. After many years without new treatment options, a number of new classes of drug therapies for diabetes have been developed. Some claim to impact cardiovascular outcome. For the most part, with the exception of metformin, the different classes of oral anti-diabetic agents are treated with equality by the clinical practice guidelines with respect to achieving A1c goal [2]. However, it may be time to consider cardiovascular outcome data when choosing a particular agent. This review summarizes the various classes of oral anti-diabetic agents with respect to cardiovascular and peripheral vascular disease data.

\section{Sulfonylureas}

Sulfonylureas have been available for more than 50 years. They stimulate insulin secretion by the pancreatic beta cell. They are relatively safe, inexpensive and well tolerated for the most part. One of the earliest studies questioning the cardiovascular risks associated with sulfonylureas was the University Group Diabetes Program (UGDP) trial [3] in which investigators recommended early termination of the tolbutamide arm of the study because of excess cardiac deaths (12.7\%) compared to placebo (4.9\%). There were many criticisms of the UGDP study including poor randomization, poor verification of cause of death, poor methodology etc., all of which led to the second larger UK Prospective Diabetes Study (UKPDS) [4] nearly thirty years later. Although the primary aim of the study was to determine the effect of intensive glycemic control, a number of additional studies were embedded into the protocol to compare the effects of different diabetes treatments on any diabetes endpoint. The effects of tighter glycemic control reduced microvascular complication rates, but effects on macrovascular disease were not statistically significant. However, there was no evidence that the sulfonylureas, glyburide and chlorpropamide, were associated with increased mortality. The controversy enjoyed a

Correspondence to: Suzanne Quinn Martinez,Clinical Associate Professor, University of Central Florida, Division of Endocrinology, Orlando Veterans Administration Hospital, USA, E-mail: Suzanne.Martinez@va.gov

Received: November 23, 2016; Accepted: November 28, 2016; Published: November 30, 2016 
brief respite. Later the discovery that sulfonylurea receptors (SUR1) on the pancreatic beta cells were different from sulfonylurea receptors on cardiacmyocytes (SUR2A) and smooth muscle cells (SUR2B) suggested that the effects of the second generation sulfonylureas such as glimepiride were more tissue specific and did not inhibit the myocardial ischemic protective effect of the $\mathrm{K}^{+}$-ATP channels in the heart and smooth muscle vasculature and offered some insight into why cardiovascular outcome data from the sulfonylureas might be contradictory [5]. This finding plus later data on other hypoglycemic agents led to a number of meta-analyses and observational studies summarized nicely in a review by Abdelmoneim, et al. [6]. In summary, the strength of the data on increased cardiovascular risk associated with sulfonylurea use remains weak.

\section{Benzoic acid derivatives}

The meglitinide drug class of oral hypoglycemics are the benzoic acid derivatives repaglinide and nateglinide. They may be thought of as the short-acting cousins of the sulfonylureas or nonsulfonylurea secretogogues. Repaglinide targets SUR1 ansd SUR 2 receptors [7] whereas nateglinide [8] has a higher selectivity for the SUR 1 receptor. There are no long-term trials evaluating cardiovascular events or mortality in patients using these agents.

\section{Metformin}

Metformin is a biguanide. Its mechanism of action is to reduce hepatic glucose output and increase glucose uptake in other tissues especially muscle thereby increasing insulin sensitivity. Metformin has also been shown to have a direct action in protecting against hyperglycemia-induced endothelial dysfunction through reduction of oxidative stress, protecting endothelial nitric oxide synthase, and reduction of mitochondrial stress [9]. The metformin alone arm of the UKPDS study revealed a $39 \%$ reduction in myocardial infarction [10]. However, when metformin was added to a sulfonlyurea to achieve glycemic control cardiovascular mortality but not risk of myocardial infarction increased. Subsequent studies have not supported this finding. In a study of more than 8000 new users of metformin and sulfonylurea [11] followed over five years, mortality rates for metformin users were $13.8 \%$ compared to $13.6 \%$ for combination users and $24.7 \%$ for sulfonylurea users. Furthermore, mortality benefit from metformin did not appear to be affected by which agent was used first.

Metformin can also add risk reduction to other classes of hypoglycemics.In two large retrospective database analyses, [12,13] metformin was also shown to reduce the incidence of congestive heart failure. Combination of metformin with a thiazolidinedione improved the reduction in risk of both heart failure and cardiovascular events [13]. In a substudy of the SCOUT trial, diet alone, metformin alone, metformin plus sulfonylurea or metformin plus insulin in diabetics at high risk of cardiovascular disease found that only diet alone or metformin alone lowered the incidence of primary cardiovascular outcomes. When combined with a DPP-4 inhibitor, the metforminDPP4 inhibitor treatment arm had 5.3 events per 1000 person years compared to 11.3 events for the metformin-sulfonylurea arm as well as a reduction in all-cause mortality [14]. Thus there is little doubt of metformin's value as a first line agent for treatment of type 2 diabetes in the absence of contraindications because of its potential for cardiovascular risk reduction.

\section{Thiazolidinediones; Cardiovascular disease}

The thiazolidinediones activate a group of nuclear receptors known as PPARs (peroxisome proliferator-activated receptors) responsible for decreasing insulin resistance, differentiating adipocytes, inhibiting VEGF-induced angiogenesis, decreasing leptin, lowering certain interleukins and exerting an anti-proliferative action. They are postulated to exert a cardiovascular benefit through their effects on endothelial function, inflammation and lipid particle makeup. Troglitazone was removed from the market due to an increased incidence of drug-induced hepatitis leaving rosiglitazone and pioglitazone. In experimental models of ischemia and reperfusion, the thiazolidinediones [15,16] reduced infarct size and improved ventricular remodeling. Then in clinical trials the drugs were shown to prevent myocardial infarction [17], reduce neointimal proliferation and restenosis after coronary stenting [18] and induce regression of atherosclerosis based on intravascular ultrasound [19].

Unfortunately, a meta-analysis of 42 trials, including DREAM [20] and ADOPT [21], on the effects of rosiglitazone on cardiovascular outcomes [22] reported an odds ratio of 1.43 for myocardial infarction and 1.64 for all-cause mortality in the rosiglitazone group. Later trials of rosiglitazone comparing intensive therapy to standard therapy including the Veterans' Affairs Diabetes Trial (VADT) [23] and the ACCORD trial [24] showed no association of rosiglitazone with increased myocardial infarction risk. The FDA restricted use of the drug in 2010. An early interim analysis of the RECORD trial showed no increase in cardiovascular outcomes in patients treated with rosiglitazone combined with metformin or sulfonylurea compared to metformin or sulfonylurea alone [25] but the results were considered inconclusive due to the low overall cardiovascular event rate. The FDA later lifted the restrictions in 2013 [26].

The BARI 2D (Bypass Angioplasty Revascularization Investigation 2 Diabetes) trial compared [27] revascularization plus intensive medical therapy versus medical therapy alone and insulin-sensitization therapy to insulin-providing therapy. Sensitization therapy was accomplished with metformin plus thiazolidinedione compared to insulin providing therapy with insulin and sulfonylurea. Although both pioglitazone and rosiglitazone were included, the predominant thiazolidinedione was rosiglitazone. Overall, the revascularization and medical therapy treatment arms had the same rate of cardiovascular events but of those assigned to the bypass grafting arm insulin sensitization provided fewer cardiovascular events, higher HDL levels, less weight gain, and less hypoglycemia suggesting insulin sensitization may be preferable in patients with type 2 diabetes and coronary artery disease.

In the PROactive trial [28], pioglitazone reduced the risk of recurrent nonfatal infarction by $28 \%$ and cerebrovascular events by $47 \%$ in patients with established cardiovascular disease although the composite primary endpoint was reduced by only $10 \%$.A randomized clinical trial comparing the safety and efficacy of rosiglitazone versus pioglitazone was completed in October 2016, but results are not yet available [29].

\section{Thiazolidinediones: Congestive heart failure}

Diabetes is an independent risk factor for congestive heart failure and left ventricular dysfunction. Thiazolidinediones, by virtue of their effects on the renal tubule, may result in fluid retention and edema and are best avoided in patients with heart failure [30]. Newer agents such as the DPP-4 inhibitors, the GLP-1 agonists and the SGLT 2 inhibitors discussed below are other options for heart failure patients. However, the thiazolidinediones appear to be safe for heart failure patients in combination with metformin [27].

\section{DPP-4 inhibitors}

The dipeptidyl peptidase-4 (DPP-4) inhibitors are a newer class of 
oral glucose lowering agent that improves glucose control by inhibiting the enzymatic degradation of incretins such as glucagon like peptide and gastric inhibitory peptide which are secreted in response to a meal [31]. These incretins then inhibit the release to glucagon resulting in increased insulin secretion and delays gastric emptying. Therefore, their effect is predominantly through reduction in postprandial glucose levels. Preclinical data, however, suggested they might also have beneficial cardiac effects through actions on other vasoactive peptides. The drug class is weight neutral, lowers postprandial lipemia, improves platelet aggregation and reduces oxidative stress [32]. Three large comparative trials, TECOS, EXAMINE and SAVOR-TIMI 53 studied the cardiovascular safety of the DPP-4 inhibitors.

The TECOS [33] trial was an FDA- mandated study to assess the cardiovascular safety of sitagliptin in the post-rosiglitazone period. TECOS was a well-designed randomized, double-blinded, placebocontrolled trial comparing sitagliptin to placebo as add-on therapy for uncontrolled type 2 diabetes in patients with known cardiovascular disease. The primary outcome was non-inferiority for cardiovascular death, nonfatal MI, nonfatal stroke or hospitalization for unstable angina. The secondary composite endpoint was superiority for cardiovascular death, nonfatal myocardial infarction, and nonfatal stroke. Sitagliptin was effective at lowering Alc but was found to be non-inferior although not superior to placebo for the primary cardiovascular endpoint. Alogliptin [34] was also shown to have a cardiovascular risk factor similar to placebo with the exception of a 3.9\% versus 3.3\% increase in the incidence of congestive heart failure.Those taking alogliptin were also significantly more likely to have their first hospital admission for heart failure (2.2 vs1.3\%). The SAVOR-TIMI [35] trial evaluated saxagliptin versus placebo. Saxagliptin was found to have similar rates of MI, stroke, cardiovascular revascularization and unstable angina compared to placebo, however, the incidence of congestive heart failure was $3.5 \%$ for saxagliptin versus $2.8 \%$ for the placebo group. As a result, the FDA issued an advisory warning regarding heart failure risk to the alogliptin and saxagliptin drug labels [36]. Later, a much larger cohort study of more than 78,000 saxagliptin and 298,000 sitagliptin users did not find an increased risk of hospitalization for heart failure among either "gliptin" user compared to other glucose-lowering "non-gliptin" agents [37]. The FDA issued an update to their original warning regarding heart failure risk and use of saxagliptin or alogliptin, but did not remove the warning [38].

Currently, the DPP-4 inhibitors are recommended as second line agents by the clinical practice guidelines [2]. For the most part they are well tolerated and can be taken without regard for food. They have few drug interactions although saxagliptin has an active CYP3A4/5 metabolite which requires dosage adjustment with concurrent use of CYP3A4/5 inhibitors. The serious side effect of pancreatitis has been anecdotally reported with all DPP -4 inhibitors [39]. Therefore, DPP-4 inhibitors should be used cautiously in patients with a history of pancreatitis [40].

\section{GLP-1 agonists}

The glucagon-like peptide-1 (GLP-1) agonists are inhibitors of GLP-1 released from the digestive tract in response to a meal. These incretin-like peptides lower glucose levels by inhibiting hepatic glucagon release, delaying gastric emptying, enhancing insulin release from the pancreas and possibly increasing beta cell mass [41]. In addition, the discovery of GLP-1 receptors in other organ systems such as the gastrointestinal tract, kidney, myocardium, vascular endothelium, and kidney leads many to hope that the drug class will have a neutral or positive effect on diabetic cardiovascular disease which has thus far been resistant to other forms of glucose-lowering therapy [42]. Wang was able to show a small reduction in systolic and diastolic blood pressure with exenatide and liraglutide [43]. Other studies have reported "modest" improvements in lipid profiles [44], reductions in various cardiovascular markers such as CRP and BNP [45] and improved left ventricular function [46].

Although some cardiovascular outcome data has been acquired from studies such as LEAD [47], HARMONY [48], and AWARD [49], the incidence of cardiac events was not the primary outcome. The ELIXA [50] trial, on the other hand, was a randomized, placebo-controlled trial designed to measure the primary outcome of cardiovascular events in lixisenatide versus placebo. The primary endpoints of cardiovascular death, myocardial infarction, stroke or hospitalization for unstable angina occurred in $13.4 \%$ of the lixisenatide group compared to $13.2 \%$ of the placebo group. Neither was there an increase in hypoglycemia, pancreatitis, nor pancreatic neoplasms. Lixisenatide was concluded to be a safe hypoglycemic agent without evidence of increased major cardiovascular events or serious side adverse effects. The Liraglutide Effect and Action in Diabetes: Evaluation of Cardiovascular Outcome Results (LEADER) trial also specifically addressed cardiovascular outcomes [51]. It showed that liraglutide was associated with significantly lower rates of death from cardiovascular causes $(4.7 \%$ in liraglutide group vs. $6.0 \%$ in placebo group), and death from any cause (8.2\% in liraglutide group compared to $9.6 \%$ in placebo group). However, the rates of nonfatal myocardial infarction, nonfatal stroke, and hospitalization for heart failure were not significantly different between the two groups.

In summary, a number of meta-analyses have looked at cardiovascular event rates in the DPP-4 inhibitors and the GLP-1 receptor agonists. However, data is inconclusive due to the small number of available trials for individual agents, different study populations and variations in study design, etc. It remains unclear which groups of patients are likely to experience benefit or harm. The GLP-1 agonist class of antidiabetics appears to be safe for patients with cardiovascular disease but is probably not the best option for patients with a history of pancreatitis, alcoholism, pancreatic neoplasm, severe hypertriglyceridemia or severe renal disease [52]. Long term safety and benefit have not been definitively established and the results of ongoing cardiovascular outcome trials should be awaited [53].

\section{Sodium-glucose cotransport 2 inhibitors}

The GLUT- 1 and 2 cotransporters are Na/ATPase-driven glucose channels responsible for reabsorption of glucose in various tissues. SGLT-2 is localized to the proximal renal tubule and accounts for up to $90 \%$ of renal glucose reabsorption. Blockade of the glucose channel by the SGLT-2 inhibitors or "flozins" leads to significant glycosuria and resultant improved glucose control. This mechanism of action does not correct the underlying altered physiology of type 2 diabetes but does offer some theoretical advantages [54]. The resultant glycosuria produces an osmotic diuresis. This effect on sodium and volume load lowers blood pressure [55] and was postulated to have ancillary beneficial cardiovascular effects [56] in addition their glucose-lowering abilities.

The EMPA-REG OUTCOME (Empagliflozin Cardiovascular Outcome Event Trial in Type 2 Diabetes Mellitus Patients) trial was a randomized, double-blinded, parallel group trial that studied all-cause mortality, cardiovascular death and hospital admissions for congestive heart failure in diabetics with high cardiovascular risk who were already receiving statins, anithypertensives and anti-platelet therapy 
[57]. Of more than 7000 patients treated, the empagliflozin group had a $3.7 \%$ versus $5.9 \%$ cardiovascular death rate, a $2.7 \%$ versus $4.1 \%$ heart failure hospitalization rate, and a $5.7 \%$ versus $8.3 \%$ all-cause mortality rate when compared to placebo. There was no significant difference in incidence of myocardial infarction or stroke. The EMPA-REG trial is currently the only completed trial of SGLT-2 inhibitor effects on cardiovascular disease outcome in type 2 diabetes however other studies remain underway. Whether this cardiovascular benefit is related to empagliflozin's pleomorphic effects or blood pressure and volume changes and whether this is a drug class effect still remain unanswered.

\section{Conclusion}

Cardiovascular disease remains the primary cause of death in type 2 diabetes. And though tighter glucose control has clearly reduced microvascular complications, the cardiovascular complications of type 2 diabetes remain frustratingly resistant to medical intervention.

When initiating monotherapy for type 2 diabetes many issues including side effect profile, cost, insurance plan, comorbidities and practitioner comfort are considered. In the absence of contraindications or intolerance, metformin remains the agent of choice for type 2 diabetes. Pioglitazone should probably not be used alone in patients with a history of heart failure but seems to be safe in combination with metformin and also has beneficial effects on lipid profiles.

The DPP-4 inhibitors appear to have neutral to beneficial effects on cardiovascular risk but show mixed results in the case of congestive heart failure with saxagliptin and alogliptin. Both still carry the FDA warnings regarding use when there is potential risk of heart failure.

The GLP-1 agonists appear to be safe in patients at high risk for cardiovascular disease but should be used cautiously if at all in patients with a history of pancreatic disease. Lixisenatide in particular has demonstrated a positive cardiovascular outcome profile. Whether this applies to the entire drug class has not yet been proven. Of the SGLT2 inhibitors, empagliflozin in particular has positive cardiovascular outcome data. Whether this applies to the entire drug class has not yet been proven.

As always, the most physiologic, least expensive, safest treatment of type 2 diabetes is diet and exercise. When lifestyle changes are no longer sufficient, practitioners now have a large armamentarium of agents from which to choose. Prescribing a particular agent for each individual should take into account the appropriate Alc goal and existing comorbidities especially cardiovascular risk. Cardiovascular disease remains a major health problem.

\section{Disclosures}

The authors have no potential conflict of interest to disclose.

\section{References}

1. American Diabetes Association.

2. Cefalu WT (2016) Standards of medical care in diabetes 2016. Diabetes Care 39: 1-119.

3. The university group diabetes program (1976) A study of the effects of hypoglycemic agents on vascular complications in patients with adult-onset diabetes. Diabetes 19: 747-780.

4. (1998) Intensive blood-glucose control with sulphonylureas or insulin compared with conventional treatment and risk of complications in patients with type 2 diabetes (UKPDS 33). UK Prospective Diabetes Study (UKPDS) Group. Lancet 352: 837-853. [Crossref]

5. Meier JJ, Gallwitz B, Schmidt WE, Mügge A, Nauck MA (2004) Is impairment of ischaemic preconditioning by sulfonylurea drugs clinically important? Heart 90: 9-12. [Crossref]

6. Abdelmoneim AS, Eurich DT, Light PE, Senior PA, Seubert JM, et al. (2015) Cardiovascular safety of sulphonylureas: over 40 years of continuous controversy without an answer. Diabetes Obes Metab 17: 523-532. [Crossref]

7. Gribble FM, Tucker SJ, Seino S, Ashcroft FM(1998) Tissue specificity of sulfonylureas studies on cloned cardiac and beta-cell K(ATP) channels. Diabetes 47: 1412-1418. [Crossref]

8. Hu S, Wang S, Dunning BE (1999) Tissue selectivity of antidiabetic agent nateglinide: study on cardiovascular and beta-cell K(ATP) channels. J Pharmacol Exp Ther 291: 1372-1379. [Crossref]

9. Kinaan M, Ding H, Triggle CR (2015) Metformin: An old drug for the treatment of diabetes but a new drug for the protection of the endothelium. Med Princ Pract 24: 401-415. [Crossref]

10. Effect of intensive blood-glucose control with metformin on complications in overweight patients with type 2 diabetes (UKPDS 34). UK prospective diabetes study (UKPDS) group. Lancet 352: 854-65. [Crossref]

11. Johnson JA, Majumdar SR, Simpson SH, Toth EL (2002) Decreased mortality associated with the use of metformin compared with sulfonylurea monotherapy in type 2 diabetes. Diabetes Care 25: 2244-2248. [Crossref]

12. Eurich DT, Majumdar SR, McAlister FA, Tsuyuki RT, Johnson JA (2005) Improved clinical outcomes associated with metformin in patients with diabetes and heart failure. Diabetes Care 28: 2345-2351. [crossref]

13. Masoudi FA, Inzucchi SE, Wang Y, Havranek EP, Foody JM, et al. (2005) Thiazolidinediones, metformin, and outcomes in older patients with diabetes and heart failure: an observational study. Circulation 111: 583-590. [Crossref]

14. Morgan CL, Mukherjee J, Jenkins-Jones S, Holden SE, Currie CJ (2014) Combination therapy with metformin plus sulphonylureas versus metformin plus DPP-4 inhibitors: association with major adverse cardiovascular events and all-cause mortality. Diabetes Obes Metab 16: 977-983. [Crossref]

15. Yue Tl TL, Chen J, Bao W, Narayanan PK, Bril A, et al. (2001) In vivo myocardia protection from ischemia/reperfusion injury by the peroxisome proliferator-activated receptor-gamma agonist rosiglitazone. Circulation 104: 2588-2594. [Crossref]

16. Shiomi T, Tsutsui H, Hayashidani S, Suematsu N, Ikeuchi M, et al. (2002) Pioglitazone, a peroxisome proliferator-activated receptor-gamma agonist, attenuates left ventricular remodeling and failure after experimental myocardial infarction. Circulation 106: 3126-3132. [Crossref]

17. Sauer WH BJ, KS (2002) Thiazolidinediones and prevention of myocardial infarction with type 2 diabetes. Circulation 106: 2777.

18. Takagi T, Yamamuro A, Tamita K, Yamabe K, Katayama M, et al. (2003) Pioglitazone reduces neointimal tissue proliferation after coronary stent implantation in patients with type 2 diabetes mellitus: an intravascular ultrasound scanning study. Am Heart $J$ 146: 366 .

19. Langenfeld MR (2005) Pioglitazone decreases carotid intima-media thickness independently of glycemic control in patients with type 2 diabetes mellitus: Results from a controlled randomized study. Circulation 111:2525-2531. [Crossref]

20. DREAM Trial Investigators, Bosch J, Yusuf S, Gerstein HC, Pogue J, et al. (2006) Effect of ramipril on the incidence of diabetes. NEngl J Med 355: 1551-1562. [Crossref]

21. Viberti G, Kahn SE, Greene DA, Herman WH, Zinman B, et al. (2002) A diabetes outcome progression trial (ADOPT): an international multicenter study of the comparative efficacy of rosiglitazone, glyburide, and metformin in recently diagnosed type 2 diabetes. Diabetes Care 25: 1737-1743. [Crossref]

22. Nissen SE, Wolski K (2007) Effect of rosiglitazone on the risk of myocardial infarction and death from cardiovascular causes. $N$ Engl J Med 356: 2457-2471. [Crossref]

23. Duckworth W, Abraira C, Moritz T, Reda D, Emanuele N, et al. (2009) Glucose control and vascular complications in veterans with type 2 diabetes. N Engl J Med 360: 129139. [Crossref]

24. Action to control cardiovascular risk in diabetes study group, Gerstein HC, Miller ME, Byington RP, Goff DC Jr, et al. (2008) Effects of intensive glucose lowering in type 2 diabetes. N Engl J Med 12: 2545-2559. [Crossref]

25. Home PD, Pocock SJ, Beck-Nielsen H, Curtis PS, Gomis R, et al. (2009) Rosiglitazone evaluated for cardiovascular outcomes in oral agent combination therapy for type 2 diabetes (RECORD): a multicentre, randomised, open-label trial. Lancet 373: 2125 2135. [Crossref] 
26. FDA Requires Removal of Certain Restrictions on the Diabetes Drug Avandia. FDA News Release. p. www.fda.gov.

27. Rutter MK, Nesto RW (2010) The BARI 2D study: A randomised trial of therapies for type 2 diabetes and coronary artery disease. Diabetes Vasc Dis Res 7: 69-72. [Crossref]

28. Charbonnel B, Dormandy J, Erdmann E, Massi-Benedetti M, Skene A, et al. (2004) The prospective pioglitazone clinical trial in macrovascular events (PROactive): can pioglitazone reduce cardiovascular events in diabetes? Study design and baseline characteristics of 5238 patients. Diabetes Care 27: 1647-1653. [Crossref]

29. Clinical Trials.gov

30. Fitchett DH, Udell JA, Inzucchi SE (2016) Heart failure outcomes in clinical trials of glucose-lowering agents in patients with diabetes. Eur J Heart Fail. [Crossref]

31. McIntosh CH, Demuth HU, Pospisilik JA, Pederson R (2005) Dipeptidyl peptidase IV inhibitors: how do they work as new antidiabetic agents? Regul Pept 128: 159-165. [Crossref]

32. Scheen AJ (2013) Cardiovascular effects of Dipeptidyl Peptidase-4 Inhibitors: From risk factors to clinical outcomes. Postgrad Med 125: 7-20.

33. Green JB, Bethel MA, Armstrong PW, Buse JB, Engel SS, et al. (2015) Effect of sitagliptin on cardiovascular outcomes in type 2 diabetes. N Engl J Med 373: 232-242. [Crossref]

34. Shimada YJ, Cannon CP, Liu Y, Wilson C, Kupfer S, et al. (2016) Ischemic cardiac outcomes and hospitalizations according to prior macrovascular disease status in patients with type 2 diabetes and recent acute coronary syndrome from the Examination of Cardiovascular Outcomes with Alogliptin versus Standard of Care trial. Am Hear J 175: 18-27. [Crossref]

35. Scirica BM, Bhatt DL, Braunwald E, Steg PG, Davidson J, et al. (2013) Saxagliptin and cardiovascular outcomes in patients with type 2 diabetes mellitus. $N$ Engl J Med 369: 1317-1326. [Crossref]

36. Drug Safety and Availability - FDA Drug Safety Communication: FDA adds warnings about heart failure risk to labels of type 2 diabetes medicines containing saxagliptin and alogliptin; Feb 11, 2014. p. www.fda.gov.

37. Toh S, Hampp C, Reichman ME, Graham DJ, Balakrishnan S, et al. (2016) Risk for hospitalized heart failure among new users of saxagliptin, sitagliptin, and othe antihyperglycemic drugs. Ann Intern Med 164: 705.

38. Drug Safety and Availability - FDA Drug Safety Communication: FDA adds warnings about heart failure risk to labels of type 2 diabetes medicines containing saxagliptin and alogliptin; April 5, 2016. Center for Drug Evaluation and Research; p. www.fda.gov.

39. Karagiannis T, Boura P, Tsapas A (2014) Safety of dipeptidyl peptidase 4 inhibitors: a perspective review. Ther Adv drug Saf 5: 138-146. [Crossref]

40. Scheen A (2013) Gliptins (dipeptidyl peptidase-4 inhibitors) and risk of acute pancreatitis. Expert Opin Drug Saf 12: 545-557. [Crossref]

41. Chon S, Gautier JF (2016) An update on the effect of incretin-based therapies on ß-cell function and mass. Diabetes Metab J 40: 99-114. [Crossref]

42. Kang YM, Jung CH (2016) Cardiovascular effects of glucagon-like peptide-1 receptor agonists. Endocrinol Metab 31: 258. [Crossref]
43. Wang B, Zhong J, Lin H, Zhao Z, Yan Z, et al. (2013) Blood pressure-lowering effects of GLP-1 receptor agonists exenatide and liraglutide: a meta-analysis of clinical trials. Diabetes, Obes Metab 15: 737-749. [Crossref]

44. Sun F, Wu S, Wang J, Guo S, Chai S, et al. (2015) Effect of glucagon-like peptide-1 receptor agonists on lipid profiles among type 2 diabetes: a systematic review and network meta-analysis. Clin Ther 37: 225-241. [Crossref]

45. Courrèges J-P, Vilsbøll T, Zdravkovic M, Le-Thi T, Krarup T, et al. (2016) Beneficial effects of once-daily liraglutide, a human glucagon-like peptide-1 analogue, on cardiovascular risk biomarkers in patients with Type 2 diabetes. Diabet Med 25: 1129 1131. [Crossref]

46. Margulies KB, Anstrom KJ, Hernandez AF, Redfield MM, Shah MR, et al. (2014) GLP-1 agonist therapy for advanced heart failure with reduced ejection fraction: design and rationale for the functional impact of GLP-1 for heart failure treatment study. Circ Heart Fail 7: 673-679. [Crossref]

47. McGill JB (2009) Insights from the liraglutide clinical development program - the liraglutide effect and action in diabetes (LEAD) studies. Postgrad Med 121: 16-25. [Crossref]

48. Pratley RE, Nauck MA, Barnett AH, Feinglos MN, Ovalle F, et al. (2014) Once-weekly albiglutide versus once-daily liraglutide in patients with type 2 diabetes inadequately controlled on oral drugs (HARMONY 7): a randomised, open-label, multicentre, noninferiority phase 3 study. Lancet Diabetes Endocrinol 2: 289-297. [Crossref]

49. Wysham C, Blevins T, Arakaki R, Colon G, Garcia P, et al. (2014) Efficacy and safety of dulaglutide added onto pioglitazone and metformin versus exenatide in type 2 diabetes in a randomized controlled trial (AWARD-1). Diabetes Care 37: 2159-2167. [Crossref]

50. Pfeffer MA, Claggett B, Diaz R, Dickstein K, Gerstein HC, et al. (2015) Lixisenatide in patients with type 2 diabetes and acute coronary syndrome. $N$ Engl J Med 373: 2247 2257.

51. Marso SP, Daniels GH, Brown-Frandsen K, Kristensen P, Mann JF, et al. (2016) Liraglutide and Cardiovascular Outcomes in Type 2 Diabetes. N Engl J Med 375: 311 322. [Crossref]

52. Egan AG, Blind E, Dunder K, de Graeff PA, Hummer BT, et al. (2014) Pancreatic safety of incretin-based drugs - FDA and EMA assessment. N Engl J Med 370: 794 797. [Crossref]

53. Wang T, Wang F, Zhou J, Tang H, Giovenale S (2016) Adverse effects of incretin-based therapies on major cardiovascular and arrhythmia events: meta-analysis of randomized trials. Diabetes Metab Res Rev 32: 843-857. [Crossref]

54. Gallo LA, Wright EM, Vallon V (2015) Probing SGLT2 as a therapeutic target for diabetes: basic physiology and consequences. Diab Vasc Dis Res 12: 78-89. [Crossref]

55. Tikkanen I, Narko K, Zeller C, Green A, Salsali A, et al. (2015) Empagliflozin reduces blood pressure in patients with type 2 diabetes and hypertension. Diabetes Care 38: 420-428. [Crossref]

56. Chilton R, Tikkanen I, Cannon CP, Crowe S, Woerle HJ, et al. (2015) Effects of empagliflozin on blood pressure and markers of arterial stiffness and vascular resistance in patients with type 2 diabetes. Diabetes Obes Metab 17: 1180-1193. [Crossref]

57. Zinman B, Wanner C, Lachin JM, Fitchett D, Bluhmki E, et al. (2015) Empagliflozin, Cardiovascular Outcomes, and Mortality in Type 2 Diabetes. $N$ Engl J Med 373: 2117 2128. [Crossref]

Copyright: (C2016 Kinaan M. This is an open-access article distributed under the terms of the Creative Commons Attribution License, which permits unrestricted use, distribution, and reproduction in any medium, provided the original author and source are credited. 